\title{
2 A Handy Novel Impedance Standard Substrate for 3 Calibration of both Horizontal and Vertical 4 Interconnects by Radio Frequency and Microwave 5 Probes
}

\author{
Bo Pu ${ }^{1}$, Taeho Kim ${ }^{2}$, Wansoo $\mathrm{Nah}^{3}$ and Jinho Joo ${ }^{4}$ \\ 1 EMC Laboratory, Missouri University of Science and Technology, Rolla, MO, 65409, USA; bobpu@ieee.org \\ 2 LG Innotek Co.,Ltd, Ansan-si, 426791, South Korea \\ 3 Department of Electrical and Computer Engineering, Sungkyunkwan University, Suwon 16419, South \\ Korea; wsnah@skku.edu \\ 4 Department of Advanced Materials Science and Engineering, Sungkyunkwan University, Suwon 16419, \\ South Korea; jinho@skku.edu \\ * Correspondence: bobpu@ieee.org
}

Received: date; Accepted: date; Published: date

\begin{abstract}
This article proposes a novel impedance standard substrate, as known as calibration kit, for the calibration using radio frequency (RF) and microwave probes, especially the through substrate which is able to calibrate not only the conventional horizontal but also the vertical interconnects on the basis of the short-open-load-through (SOLT) standardized calibration approach. In the measurement of a vertical connection where the probing ports are located on the opposite surfaces of devices, a location change or rotate with the probes is required after the calibration based on the conventional coplanar kit which only allows the probing in the same plane. The location change or rotation of the probes and corresponding connected cables inevitably affects the precision of the calibration since the probes and cables are sensitive to the surroundings. Our proposed calibration kit can overcome the aforementioned shortcomings, and the comparison of calibrated results between conventional and proposed through impedance standard substrate is demonstrated. Validation of the proposed kit based on the coplanar meander lines and vertical couplings by via arrays is also discussed.
\end{abstract}

Keywords: Calibration; coupling coefficient; impedance standard substrate; radio frequency/microwave probes; short-open-load-through, vertical through interconnect;

\section{Introduction}

The semiconductor technology is going to the More than Moore's era where the integration of integrated circuits (ICs) in three dimensional directions is widely used [1]. Vertical connection such as via in printed circuit board (PCB) or through silicon via (TSV) in 2.5D silicon interposer or 3D chips is necessary for the signal propagation in diverse hierarchical levels as shown in Figure 1 [2] [5]. Vector Network Analyzer (VNA) is the equipment to test the characteristics of components in electronic devices, and verify design simulations to make sure the high speed signal propagates properly. Usually, a VNA is to measure the network parameters of the electrical networks such as 

easy to measure at high frequencies [6].
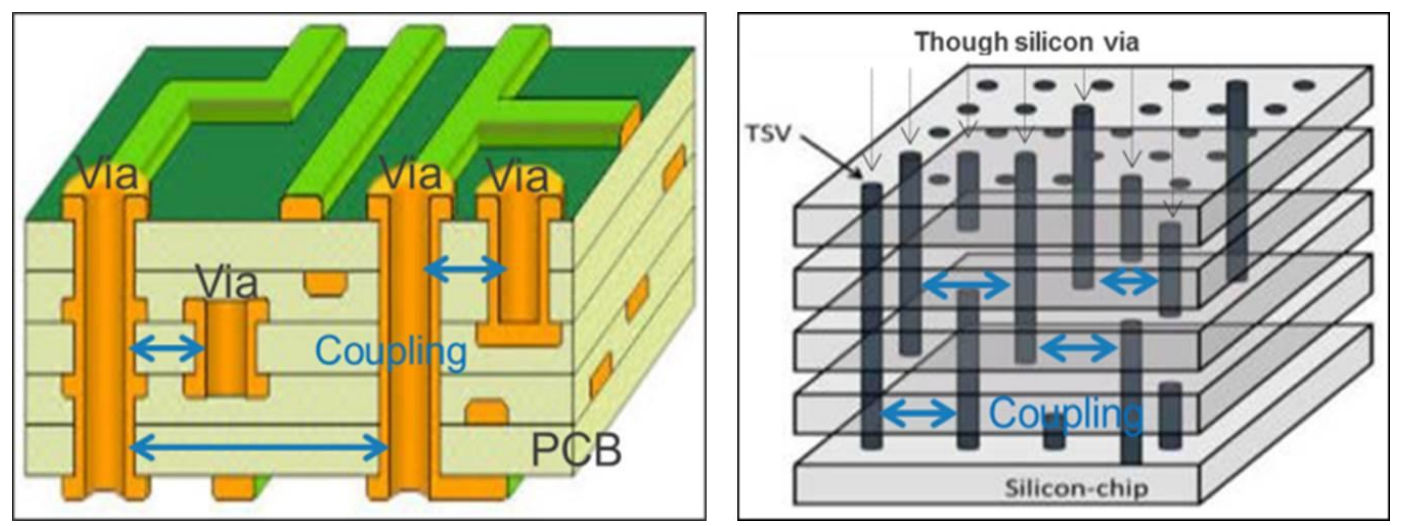

Figure 1. Vertical connection in PCB and 3D integrated circuits.

To ensure the accuracy of measurement by VNA, a calibration is required before error-corrected tests are performed. The common calibration algorithm used for multi-port measurement is known as short-open-load-through (SOLT) or Through-Reflect-Line (TRL) methods [7][8]. The most common calibration method $-S$ OLT is generally used as they are easily manufactured, and can cover a wide range of magnitude and phase. It typically utilizes three impedances which are short, open, and load, and one transmission standard, as named through/thru, to define the calibrated reference plane. In many non-coaxial situations, SOLT calibration standard is difficult, if not impossible, to build. The non-availability of calibration standards in proprietary or unique coaxial connectors also benefits from the TRL calibration method. No matter which method we choose, an appropriate through design is crucial to the performance of calibration.

In a calibration process, the various error factors, which are used to describe a VNA, result in a system of equations using actual and measured $S$-parameters to obtain the error terms of VNA model. Actual S-parameters are achieved with error-free measurement equipment such as VNA, and measured $S$-parameters are obtained during the calibration. In a general, measured $S$-parameters include the factors of both device under test (DUT) and VNA, while actual $S$-parameters describe the characteristics of DUT only. A calibration method is a procedure to minimize the difference between these two parameters, and defines how we use the calibration standards to obtain enough system information to solve for our error correction models.

Appropriate calibration algorithm on the basis of impedance standard substrate, also known as calibration kit, guarantees the establishment of error correction model in a right way. The short, open and load kit stands for one-port components that canonically have reflection coefficients of -1, 1 and 0 . These three components are measured to solve for the 3-term one-port model during vector error correction (VEC). Most transmission parameter for error models are generated in through case.

A conventional horizontal Thru impedance standard substrate is illustrated in Figure 2 [9]. The metal surface of kit is coplanar type, and only has one side for probing. In the measurement of a vertical connection where the probing ports are located on the opposite surfaces of devices, a 
location change or rotate with the probes is required after the calibration based on the conventional coplanar kit since it only allows the probing in the same plane Figure 3. The location change or rotate of the probes and corresponding connected cables inevitably affects the precision of the calibration since the probes and cables are sensitive to the surroundings. As a result, calibration kit, especially, the import thru case for calibrations in both horizontal and vertical connections, is required.

In this paper, we proposed a novel calibration kit for the calibration of interconnects in both horizontal and vertical connections. Section 2 introduced the theoretical background of through and the proposed novel through structure as well as its validation. Implementation of the proposed kit on the horizontal and vertical interconnects is discussed in Section 3. Ultimately, a conclusion is obtained in Section 4.

\section{3}

\section{(1)} 86

Figure 2. A conventional horizontal coplanar impedance standard substrate for short, thru, and load.
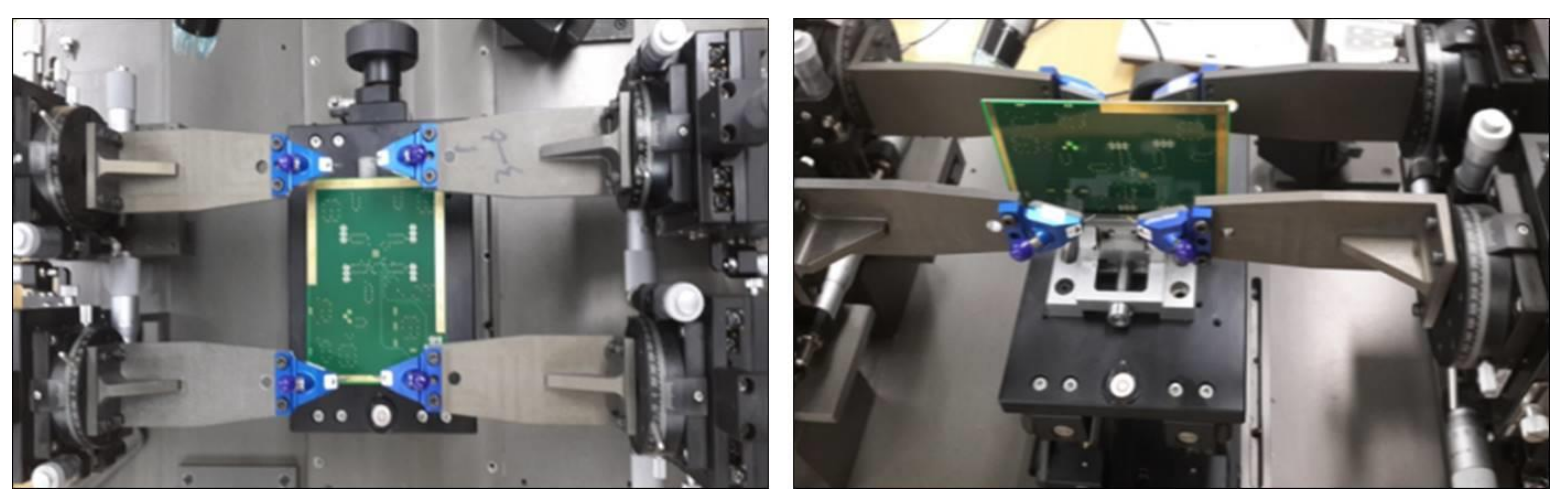

Figure 3. Probing locations in horizontal (left) and vertical interconnects (right) in the measurement by flexible probe system.

\section{Theoretical Background of Through Case and Proposed Structure for Calibration}

The characteristic of a transmission line is described by the Telegrapher's equation in (1). The voltage and current propagates along the electrical transmission line with distance and time is represented by a pair of coupled, linear partial differential equations.

$$
\begin{aligned}
& \frac{\partial v(z, t)}{\partial z}=-R i(z, t)-L \frac{\partial i(z, t)}{\partial t} \\
& \frac{\partial i(z, t)}{\partial z}=-G v(z, t)-C \frac{\partial v(z, t)}{\partial t}
\end{aligned}
$$


The Telegrapher's equations is changed to (2) in the sinusoidal steady-state, where $w$ is the angular frequency of the steady-state wave. A wave equation for voltage or current is obtained in (3), where $\gamma$ is the propagation constant, and can be written by the attenuation constant $\alpha$ and phase constant $\beta$ as well as the lumped parameters RLGC as shown in (4)[10]..

$$
\begin{aligned}
& \frac{d V(z)}{d z}=-(R+j w L) I(z) \\
& \frac{d I(z)}{d z}=-(G+j w C) V(z)
\end{aligned}
$$

98

$$
\begin{aligned}
& \frac{d^{2} V(z)}{d z^{2}}-\gamma^{2} V(z)=0 \\
& \frac{d^{2} I(z)}{d z^{2}}-\gamma^{2} I(z)=0
\end{aligned},
$$

99

$$
\gamma=\alpha+j \beta=\sqrt{(R+j w L)(G+j w C)}
$$

100

101

102

103

104

A transmission line with characteristic impedance $Z_{c}$, propagation constant $\gamma$ and length $l$ can be converted to a two-port network. The $A B C D$ matrix of the network is given as (5). At the same time, there is a well-known relationship between $S$-parameters and $A B C D$ matrix as described in (6).

$$
\left[\begin{array}{ll}
A & B \\
C & D
\end{array}\right]=\left[\begin{array}{ll}
\cosh \gamma l & Z_{c} \sinh \gamma l \\
\frac{\sinh \gamma l}{Z_{c}} & \cosh \gamma l
\end{array}\right]
$$

$$
\begin{array}{ll}
S_{11}=\frac{A+B / Z_{0}-C Z_{0}-D}{A+B / Z_{0}+C Z_{0}+D} & S_{12}=\frac{2(A D-B C)}{A+B / Z_{0}+C Z_{0}+D} \\
S_{21}=\frac{2}{A+B / Z_{0}+C Z_{0}+D} & S_{22}=\frac{-A+B / Z_{0}-C Z_{0}+D}{A+B / Z_{0}+C Z_{0}+D}
\end{array}
$$

Usually, the impedances of both input and output equal to reference impedance $Z_{0}$, we can make a relationship between the terms of $\mathrm{ABCD}$ matrix and S-parameters as in (7).

$$
\begin{aligned}
& A=\frac{1-S_{11}^{2}+S_{21}^{2}}{2 S_{21}^{2}} \\
& B=\frac{\left(1+2 S_{11}+S_{11}^{2}-S_{21}^{2}\right) Z_{0}}{2 S_{11}} \\
& C=\frac{\left(1-2 S_{11}+S_{11}^{2}-S_{21}^{2}\right)}{2 S_{11} Z_{0}} \\
& D=\frac{1-S_{11}^{2}+S_{21}^{2}}{2 S_{21}^{2}}
\end{aligned}
$$

Based on (5) and (6), the $S$-parameters are modified as the expression of characteristic and reference impedance as well as propagation constant as in (8). 


$$
\left[\begin{array}{ll}
S_{11} & S_{12} \\
S_{21} & S_{22}
\end{array}\right]=\frac{1}{D_{1}}\left[\begin{array}{cc}
\left(Z_{C}^{2}-Z_{0}^{2}\right) \sinh \gamma l & 2 Z_{C} Z_{0} \\
2 Z_{C} Z_{0} & \left(Z_{C}^{2}-Z_{0}^{2}\right) \sinh \gamma l
\end{array}\right]
$$

109

110

111

112

Where $D_{1}=2\left(Z_{C} Z_{0}\right) \cosh \gamma l+\left(Z_{C}^{2}+Z_{0}^{2}\right) \sinh \gamma l$.

Then, we take an operation with terms of $S$-parameters, and use impedance and $S$-parameters to replace the propagation constant and length from (9) to (12). Finally, only the expression of the characteristic impedance is left using the reference impedance and $S$-parameters in (12).

$$
\begin{gathered}
\frac{S_{11}}{S_{21}}=\frac{\left(Z_{C}^{2}-Z_{0}^{2}\right) \sinh \gamma l}{2 Z_{C} Z_{0}}=\frac{\left(Z_{C}^{2}-Z_{0}^{2}\right) \frac{Z_{0}}{Z_{C}} \frac{\left(1+S_{11}\right)^{2}-S_{21}^{2}}{2 S_{21}}}{2 Z_{C} Z_{0}} \\
Z_{C}^{2}=\frac{Z_{0}^{2}\left(S_{21}^{2}-\left(1+S_{11}\right)^{2}\right)}{4 S_{11}-1-2 S_{11}+S_{11}^{2}+S_{21}^{2}}=Z_{0}^{2} \frac{\left(1+S_{11}\right)^{2}-S_{21}^{2}}{\left(1-S_{11}\right)^{2}-S_{21}^{2}} \\
\left(\frac{Z_{C}}{Z_{0}}\right)^{2}=\frac{\left(1+S_{11}\right)^{2}-S_{21}^{2}}{\left(1-S_{11}\right)^{2}-S_{21}^{2}}
\end{gathered}
$$

113

$$
Z_{C}(\omega)=Z_{0} \sqrt{\frac{\left(1+S_{11}\right)^{2}-S_{21}^{2}}{\left(1-S_{11}\right)^{2}-S_{21}^{2}}}
$$

114

115

116

Substitute the characteristic impedance $Z_{c}$ into the insertion loss term $S_{21}$, and an equation for propagation constant, length and S-parameters is obtained in (13). The hyperbolic cosine of the propagation constant and length is able to be further written as (14).

$$
\begin{aligned}
S_{21} & =\frac{2 Z_{C} Z_{0}}{2\left(Z_{C} Z_{0}\right) \cosh \gamma l+\left(Z_{C}^{2}+Z_{0}^{2}\right) \sinh \gamma l} \\
& =\frac{2 Z_{C} Z_{0}}{2\left(Z_{C} Z_{0}\right) \cosh \gamma l+\left(Z_{C}^{2}+Z_{0}^{2}\right) \frac{Z_{0}}{Z_{C}} \frac{\left(1+S_{11}\right)^{2}-S_{21}^{2}}{2 S_{21}}} \\
& =\frac{2}{2 \cosh \gamma l+\frac{\left(Z_{C}^{2}+Z_{0}^{2}\right)}{\left(Z_{C}^{2}\right)} \frac{\left(1+S_{11}\right)^{2}-S_{21}^{2}}{2 S_{21}}} \\
& =\frac{2 \cosh \gamma l+\left(1+\left(\frac{Z_{0}}{Z_{C}}\right)^{2}\right) \frac{\left(1+S_{11}\right)^{2}-S_{21}^{2}}{2 S_{21}}}{\cosh \gamma l+\frac{2+2 S_{11}^{2}-2 S_{21}^{2}}{4 S_{21}}} \\
& =\frac{2}{2}
\end{aligned}
$$




$$
\cosh \gamma l=\frac{1}{S_{21}}-\frac{1+S_{11}^{2}-S_{21}^{2}}{2 S_{21}}=\frac{1-S_{11}^{2}+S_{21}^{2}}{2 S_{21}}
$$

118

Ultimately, the propagation constant is able to be derived by the $S$-parameters and length of transmission network as in (15),

$$
\gamma=\frac{1}{l} \cosh ^{-1}\left(\frac{1-S_{11}^{2}+S_{21}^{2}}{2 S_{21}^{2}}\right)
$$

Where the unit of symbols are $\gamma$ : propagation constant $(1 / \mathrm{m}) " ; \alpha$ : "Attenuation constant $(\mathrm{Neper} / \mathrm{m}) " ; \quad \beta$ : "Phase constant $(\operatorname{Rad} / \mathrm{m}) " ; l^{\prime \prime}$ length of transmission line $(\mathrm{m})$ ".

An ideal thru in impedance standard substrate is a transmission line with zero-length, no reflection and pure transmission. However, a through in the realistic scenario has a finite length, and reflection is caused by impedance mismatch, and loss for transmission. The through calibration standard is used for obtaining the transfer characteristics between two ports. Electrical length of a through affects the phase of propagated signal, and generates a bias in the phase. The phase bias is able to be compensated in the calibration by a value of propagation constant for the through, which generates the phase bias exactly. Compensated propagation constant is derived from the measured $S$-parameters of the Thru line on the basis of (15).

The proposed through has two sides, with the same pattern in each. Each surface is designed as a symmetrical structure, and three vias, two grounds and one signal, were used to connect both sides as illustrated in Figure 4. Fabricated calibration kit with SOLT impedance standard substrates is shown in Figure 5, where the Thru is on the basis of proposed one, and short, open and load are same with conventional kit.

Detailed calibration process for diverse probing types was illustrated in Figure 6. Both Signal-Ground (SG)/Ground Signal (GS) (upper-left) and SG/SG probes (upper-right) can be used for the calibration in the same side. Similarly, the SG/GS (lower-left) and SG/SG probes (lower-right) are able to be implemented in the calibration for two opposite sides of the calibration kit.

The through was designed with FR-4 material as the substrate and copper as the transmission line, with vertical and horizontal lengths of $1.66 \mathrm{~mm}$ as in Figure 4. Although the original design is proposed for calibration of four ports, in this article, we would like to discuss its implementation on two ports, and validate the accuracy from DC to $8 \mathrm{GHz}$, which is already enough for the calibration of a large range of the RF and microwave components.

Single-ended characteristic impedance of the proposed through is $51 \sim 53 \Omega$ in the frequency range from $300 \mathrm{kHz}$ to $8.5 \mathrm{GHz}$ as shown in Figure 7 for SG/SG and SG/GS in both horizontal and vertical directions. The tolerance of the manufacturing process is $200 \mathrm{um}$ for via diameter, and 100 um for pad size.
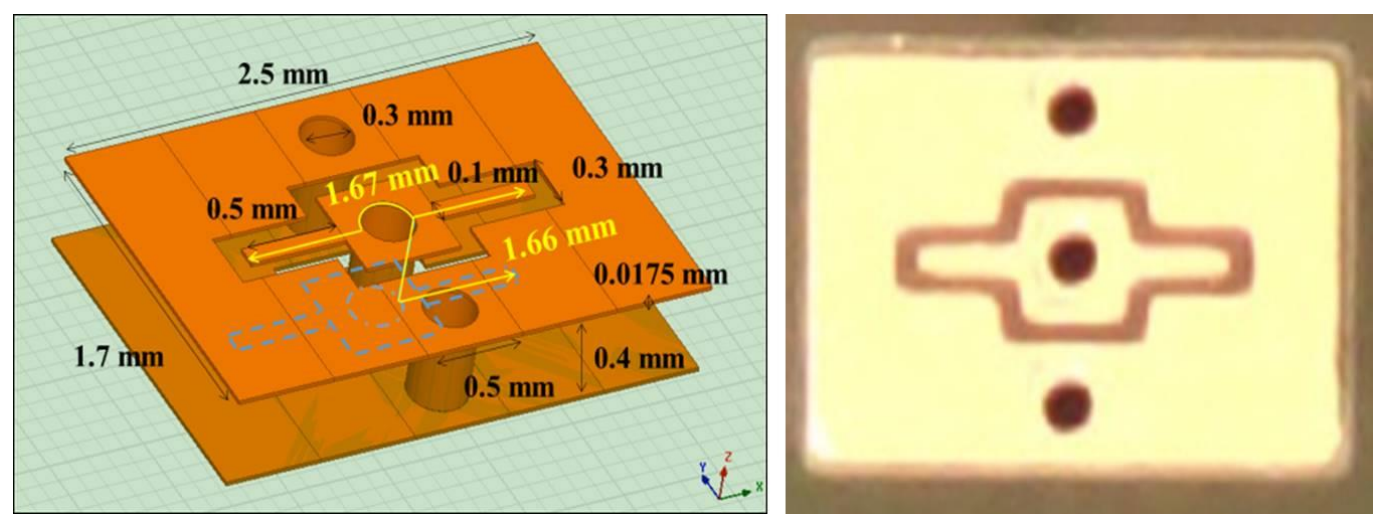

Figure 4. Proposed through structure for calibrations in both horizontal and vertical interconnectors. 

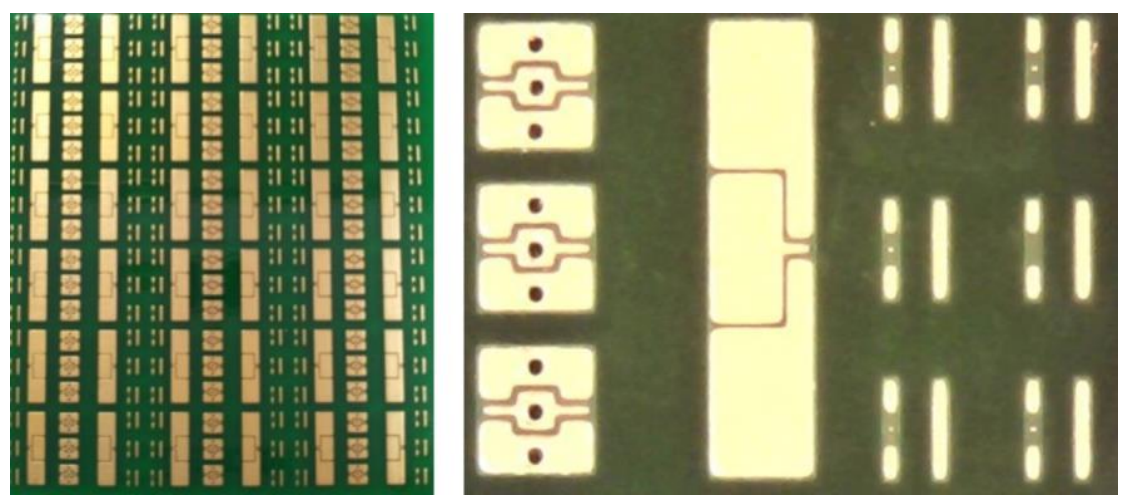

Figure 5. Fabricated calibration kit with proposed Thru, short, open, load impedance standard substrates.

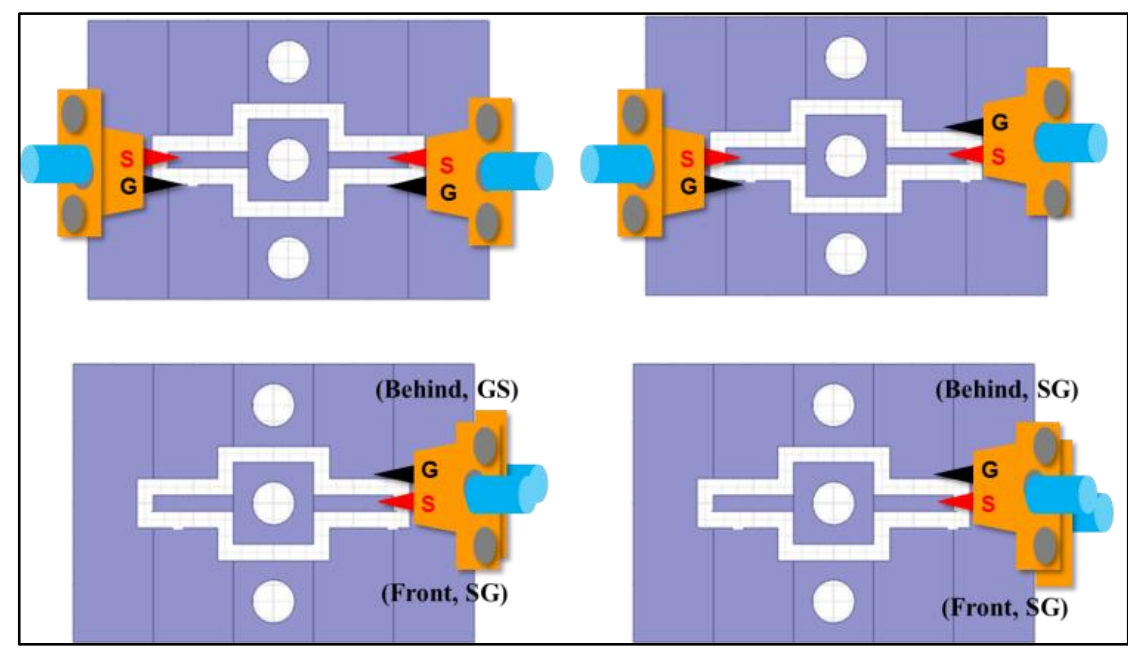

Figure 6. Calibration for diverse probing types (horizontal probing on the same plane for upper two cases, and vertical probing on different planes for lower two cases).
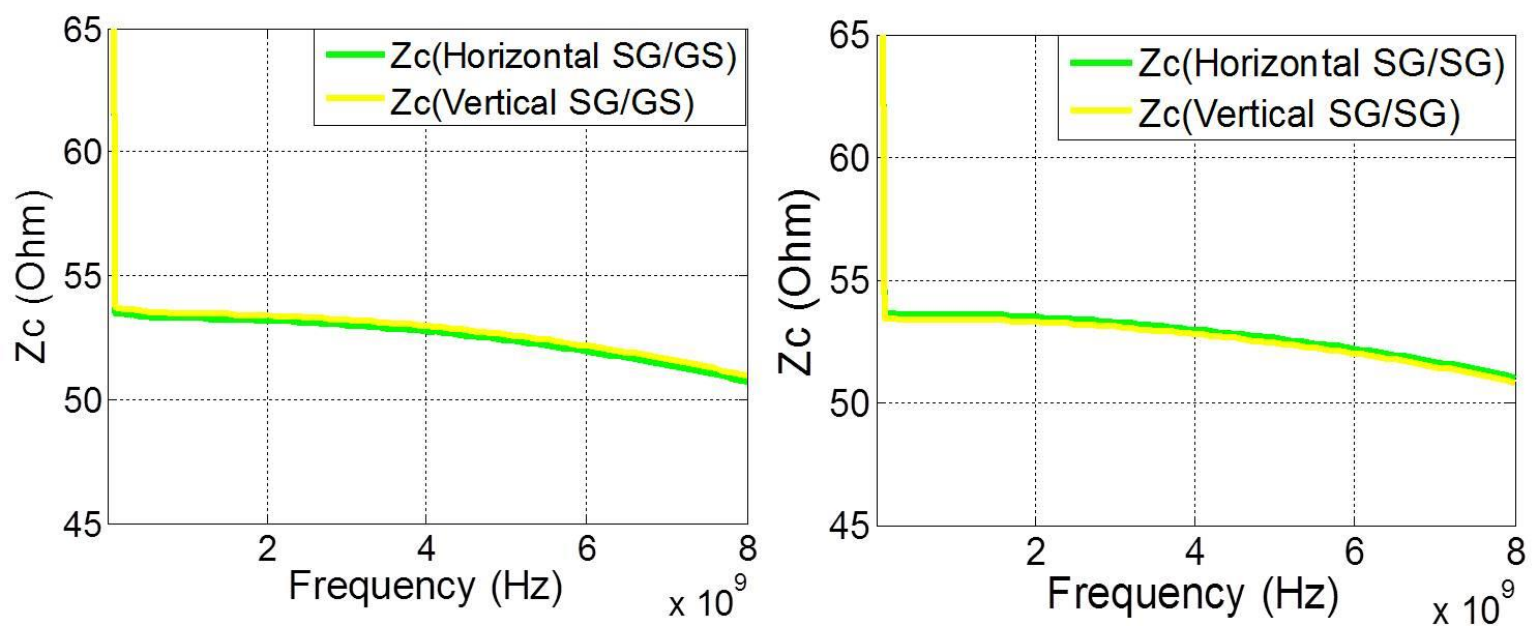

Figure 7. Characteristic Impedance for the proposed Thru in a wide frequency range.

Comparison of reflection loss for the proposed and conventional commercial Cascade calibration kits is illustrated in Figure 8. The return loss of proposed Thru is higher than that in commercial kit, but it is still acceptable (less than -20dB). Furthermore, the reflection in proposed one is more stable than the commercial kit in both magnitude and phase. In those cases, VNA was 


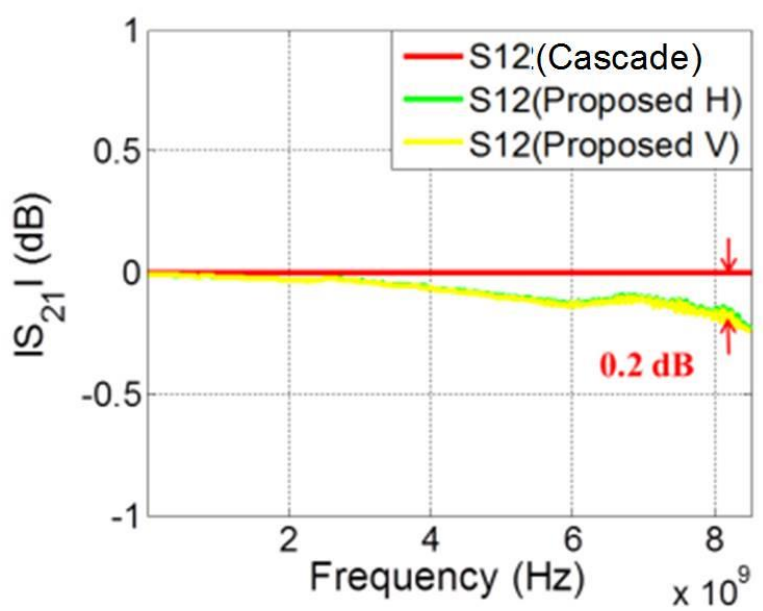
difference in phase from $300 \mathrm{kHz}$ to $8.5 \mathrm{GHz}$.

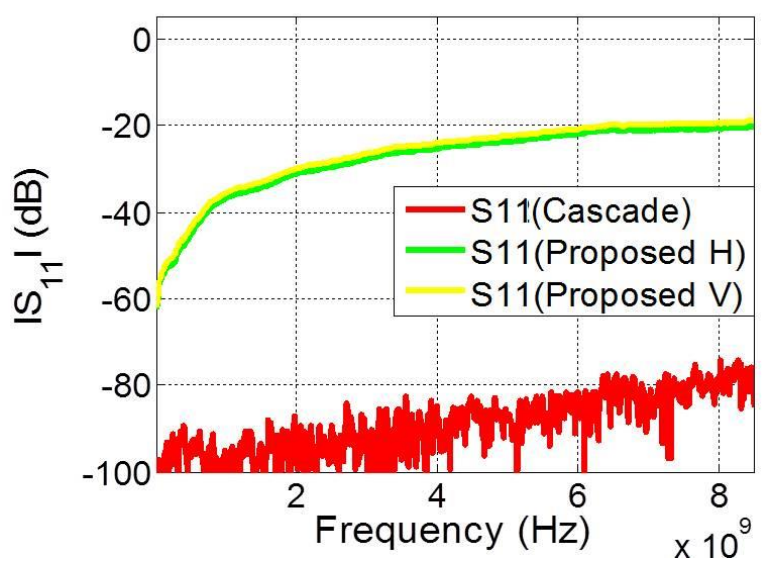

calibrated using SG-SG Cascade calibration kit shown in Figure 2. As shown in Figure 9, insertion loss of the proposed Thru by SG/GS probing has a maximum $0.2 \mathrm{~dB}$ difference in magnitude and $20^{\circ}$

Notice that the degree of $S_{11}$ for Cascade case fluctuates very much because of so small magnitudes of S11. Since the probes were calibrated on the basis of the Cascade Thru case, the insertion loss of Cascade Thru definitely returns to 0 when we measure the loss of the two Thru cases after calibration. Furthermore the large phase difference in S21 is due to the difference in lengths: $650 \mu \mathrm{m}$ for SG/GS case in Cascade while 1670um for SG/GS in our proposed one. Lengths of Thru are intentionally designed around 1670 for both SG/GS and SG/SG in our proposed kit, and it could be a merit which maintains the same propagation bias in different probing scenarios while commercial one cannot (650um for SG/GS and 1650um for SG/SG). In SG/SG case, return loss has the similar result with that in SG/GS case as in Figure 10, and differences of magnitude and phase of insertion loss are approximately equal as shown in Figure $\mathbf{1 1}$ because the lengths of Thru are also the same in both commercial Cascade and our proposed Thru cases.

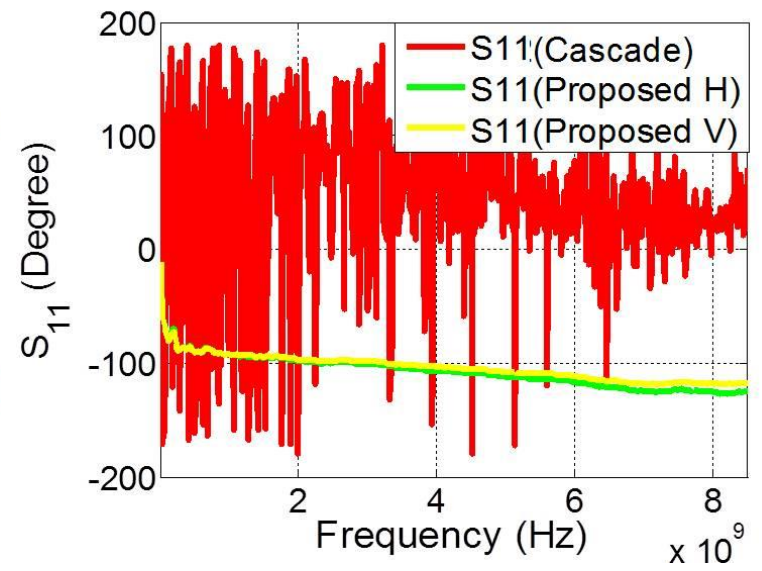

Figure 8. Comparison of reflection loss of conventional (cascade) and proposed Thru measured by SG-GS probe type after calibration based on conventional kit.

Figure 9. Comparison of insertion loss of conventional (cascade) and proposed Thru measured by

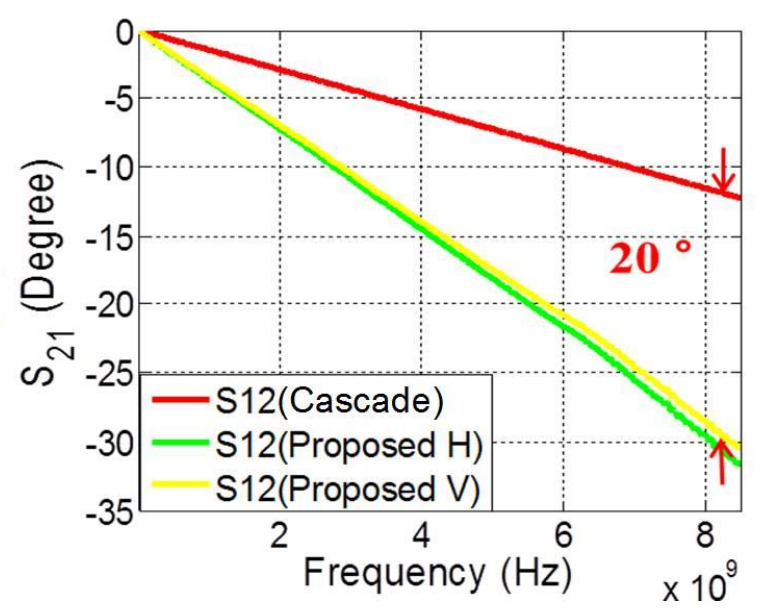



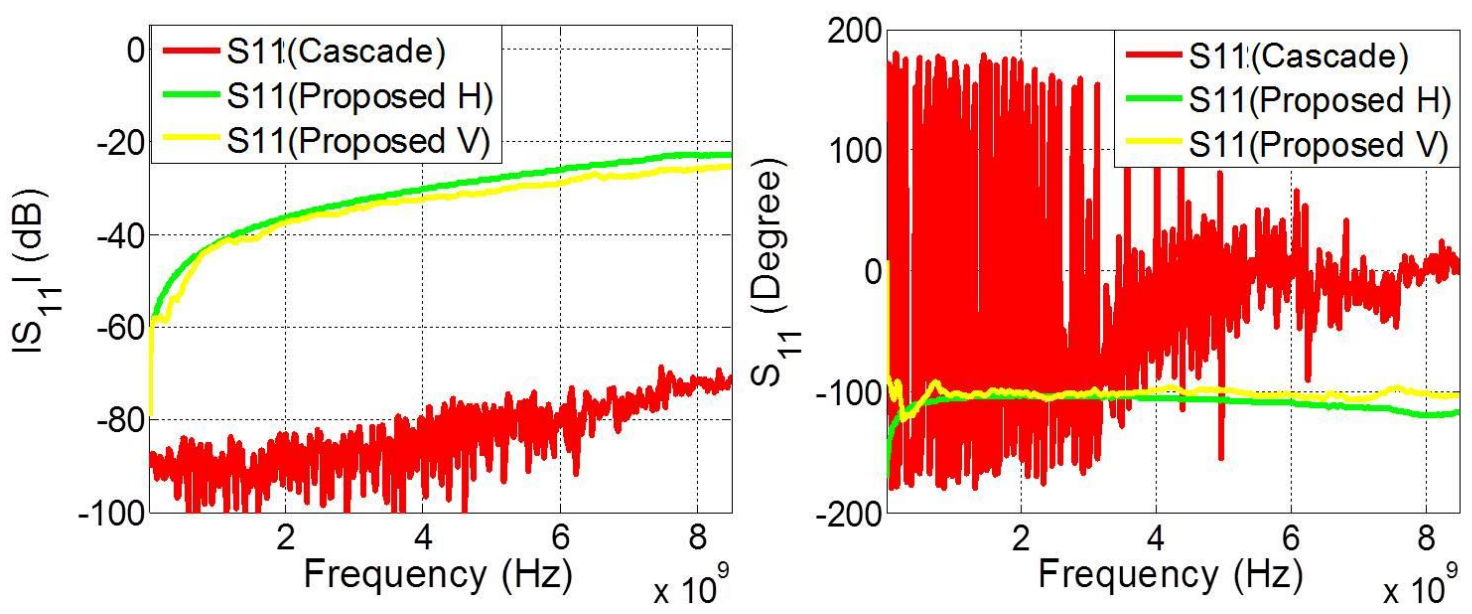

Figure 10. Comparison of reflection loss of conventional (cascade) and proposed Thru measured by SG-SG probe type after calibration based on conventional kit.

189
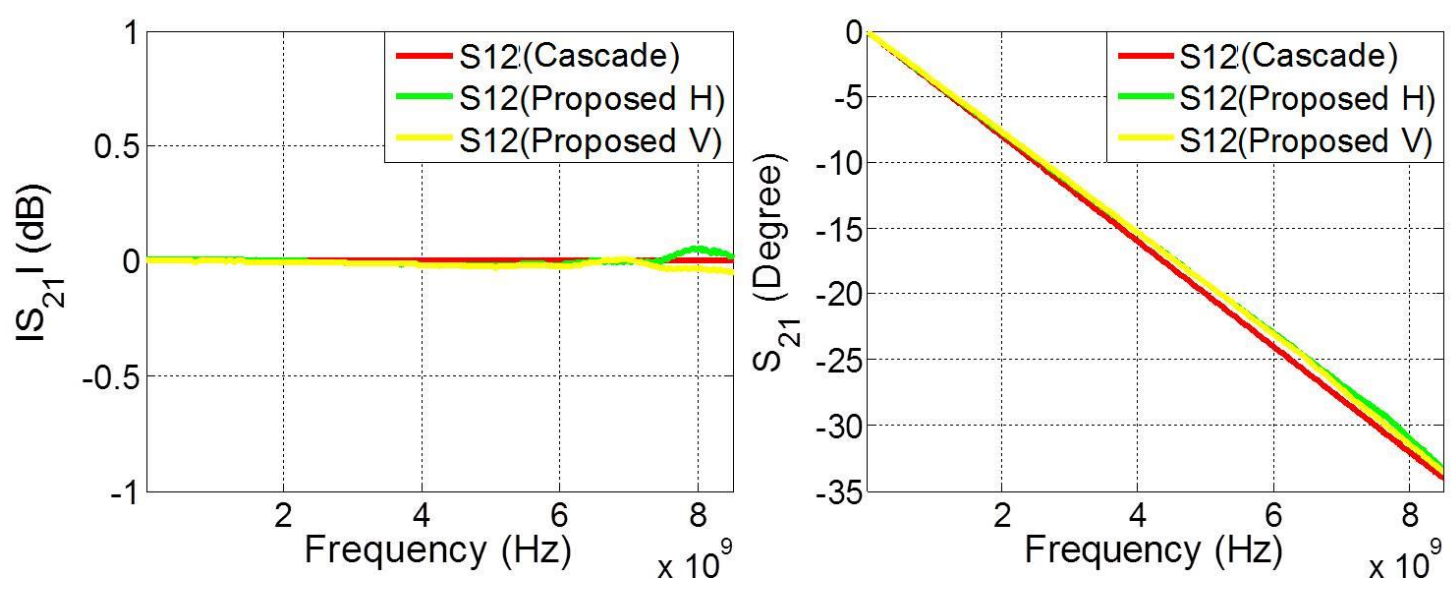

Figure 11. Comparison of insertion loss of conventional (cascade) and proposed Thru measured by SG-SGprobe type for after calibration based on conventional kit.
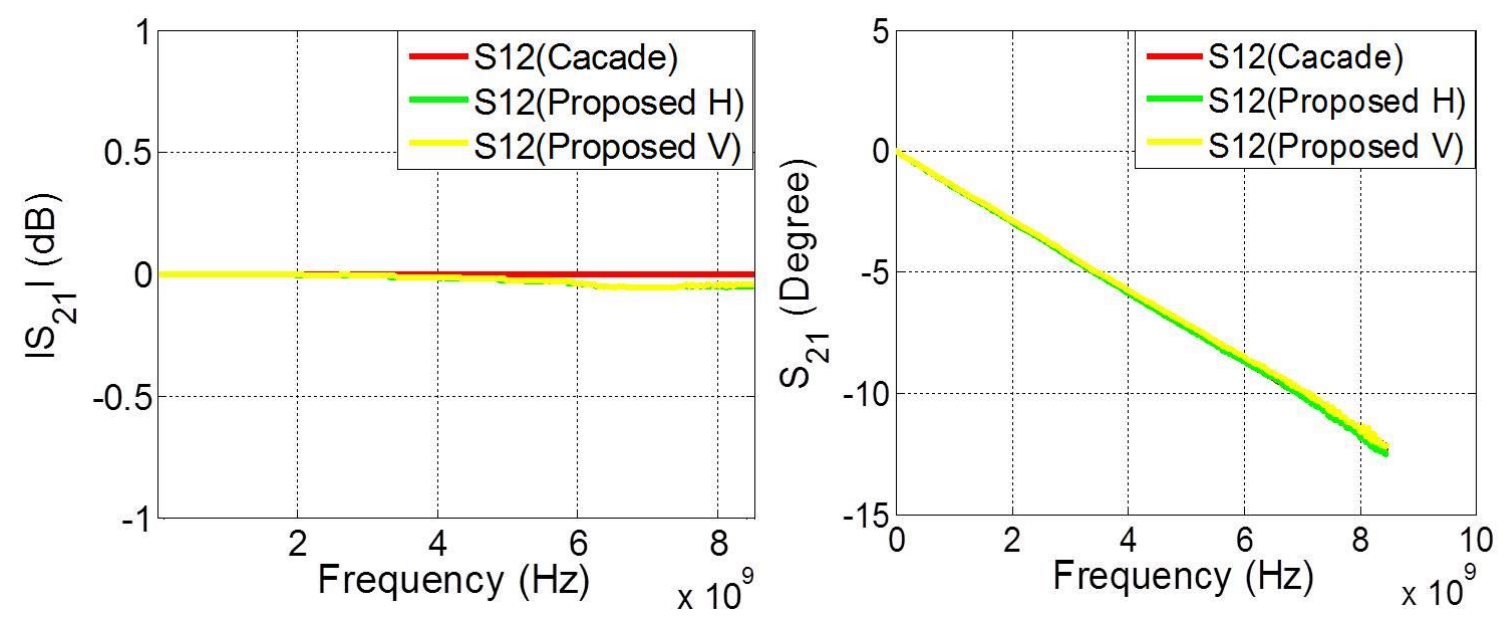

Figure 12. Results of Thru characteristics (Insertion loss-S21 magnitude and phase using an SG/GS probe) compensated by a propagation constant of the Thru kit.

The effective dielectric permittivity, $\varepsilon_{-}$eff, of proposed Thru is quite difficult to be estimated due to the geometrical complexity, however, analytical equations was used to calculate $\varepsilon \_$eff for the 
simple coplanar waveguide shape in this proposed Thru structure. The approximated $\varepsilon_{-}$eff is calculated as 3.0, and then the phase difference $\beta=\omega \sqrt{ }\left(\varepsilon \_\right.$eff $) \quad 1 / \mathrm{c}=\llbracket 30 \Omega \wedge_{\mathrm{O}}$ at $8 \mathrm{GHz}$, which is consistence with the measurement result shown in Figure 11.

As we mentioned, there is a phase difference due to diverse lengths of Thru for SG/GS probing as shown in Figure 9. The phase bias is able to be compensated if we know the propagation delay of the length. Compensated insertion loss for SG/GS probing is illustrated in Figure 12, and $0.2 \mathrm{~dB}$ of magnitude and $(20 \Omega \wedge$ of phase for the difference of insertion loss are diminished.

Generally, a VNA has an option for the compensation of phase difference. The option is expressed as a group delay calculated as $T_{g}=l \frac{d \beta}{d w}$, and the result for commercial Cascade and proposed Thru cases is illustrated in Figure 13. Moreover, values of the offset group delay used in our compensation for two Thru cases are listed in Table 1.

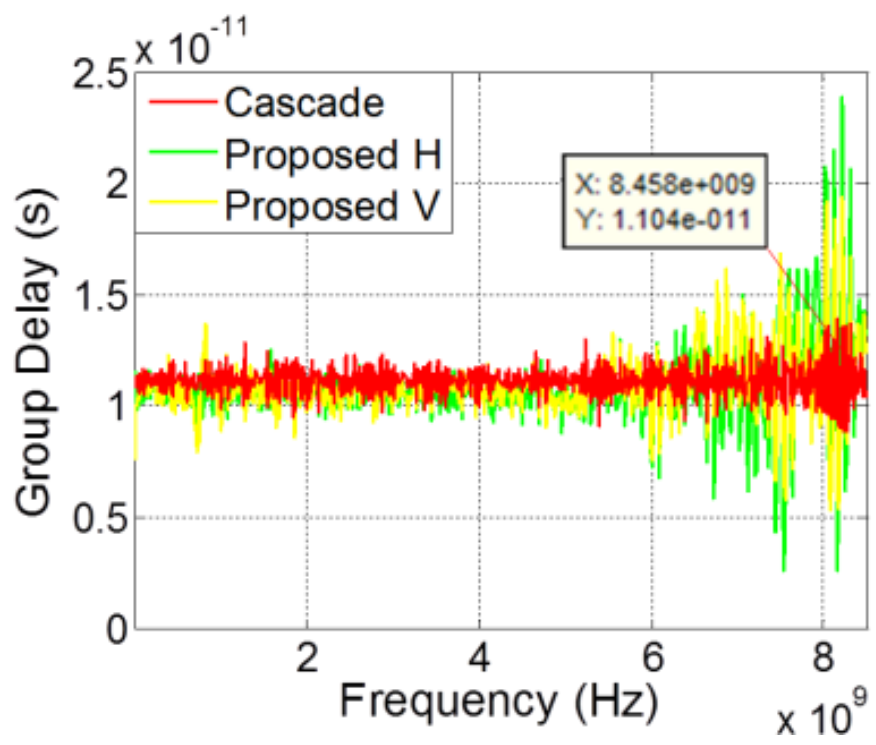

Figure 13. Group delay for commercial Cascade and proposed Thru impedance standard substrate.

Table 1. Group delay for commercial and proposed Thru cases in diverse probing scenarios.

\begin{tabular}{ccc}
\hline & \multicolumn{2}{c}{ Group Delay (ps) } \\
\hline Probing Type & SG/GS & SG/SG \\
\hline Commercial Thru & 6 & 11.1 \\
Proposed Thru & 11.1 & 11.1 \\
\hline
\end{tabular}

\section{Implementation and Validation of Proposed Thru Impedance Standard Substrate}

In order to validate the proposed Thru impedance standard substrate, we performed the measurement on the basis of designed symmetrical meander lines. Symmetrical meander lines have the characteristic of dense structure with high coplanar inductive coupling, and is suitable for the signal-ground probing. The design and fabrication of meander lines were discussed in [11], DUT here has a width of $1.25 \mathrm{~mm}$, a length of $5 \mathrm{~mm}$, and 17 turns. The PCB is one-sided and is composed of FR-4 and copper. Calibrations by commercial and proposed impedance standard substrates were taken first, and then probes were located on the ports of meander lines to characterize the $S$-parameters. The characteristics of measured meander lines on the basis of two SG/GS calibrations are illustrated in Figure 15 and Figure 16. Result from the commercial calibration kit and proposed one match each other well from DC to $8 \mathrm{GHz}$ for both magnitude and phases as return loss in Figure 15 and insertion loss in Figure 16. The same good agreement for results from two calibration ways by SG/SG probes is also found in Figure 17 and Figure 18. The validation of proposed calibration kit can achieve the same performance up to $8 \mathrm{GHz}$ as that by commercial one, but keeps a merit for both horizontal and vertical calibration. As a result, the proposed kit is appropriate for accurate and handy calibration. 


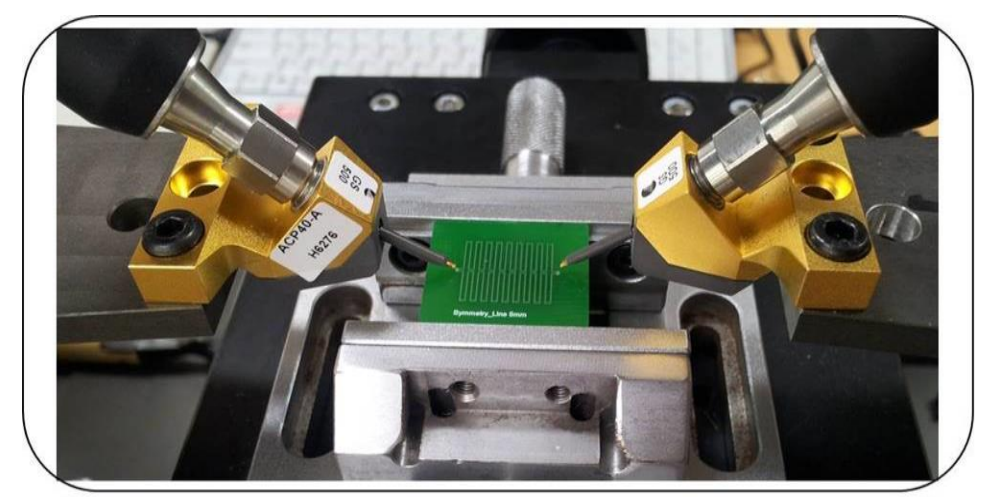

Figure 14. S-parameter measurement for meander lines after calibration.
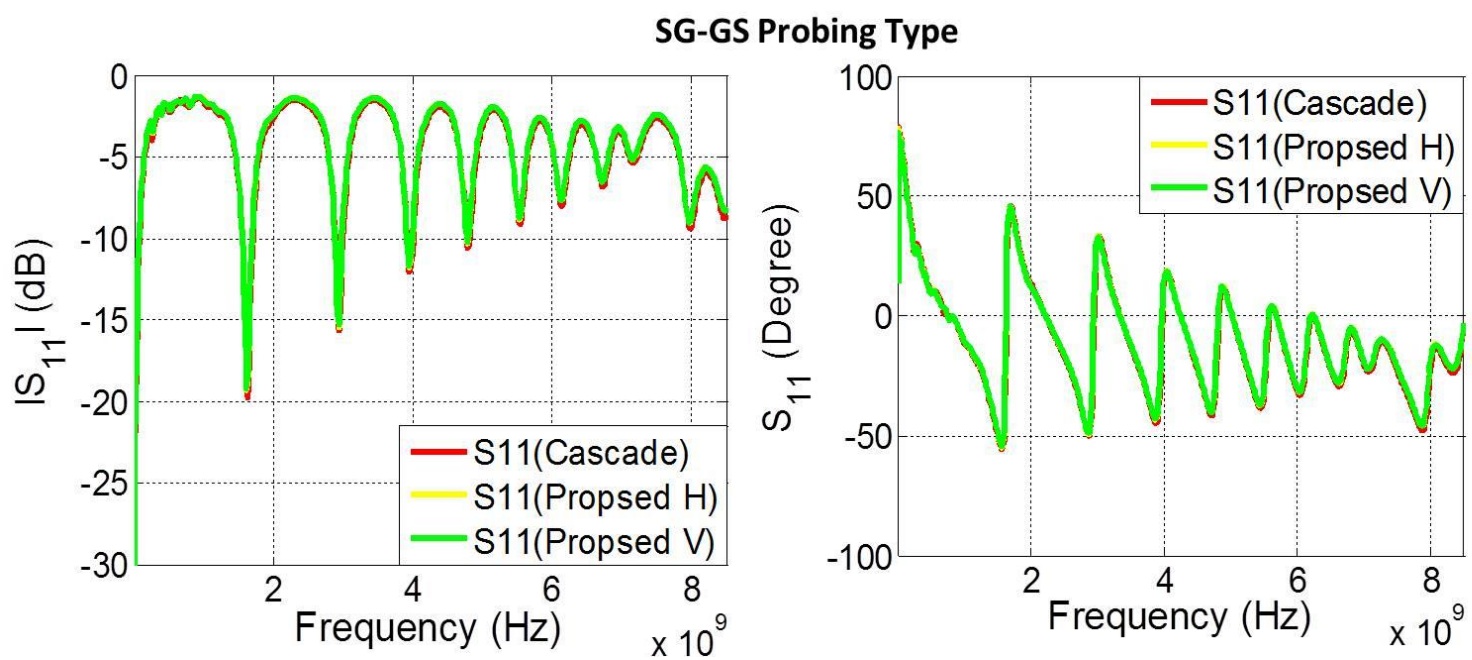

Figure 15. Return loss of meander lines measured after SG/GS calibration by commercial Cascade and
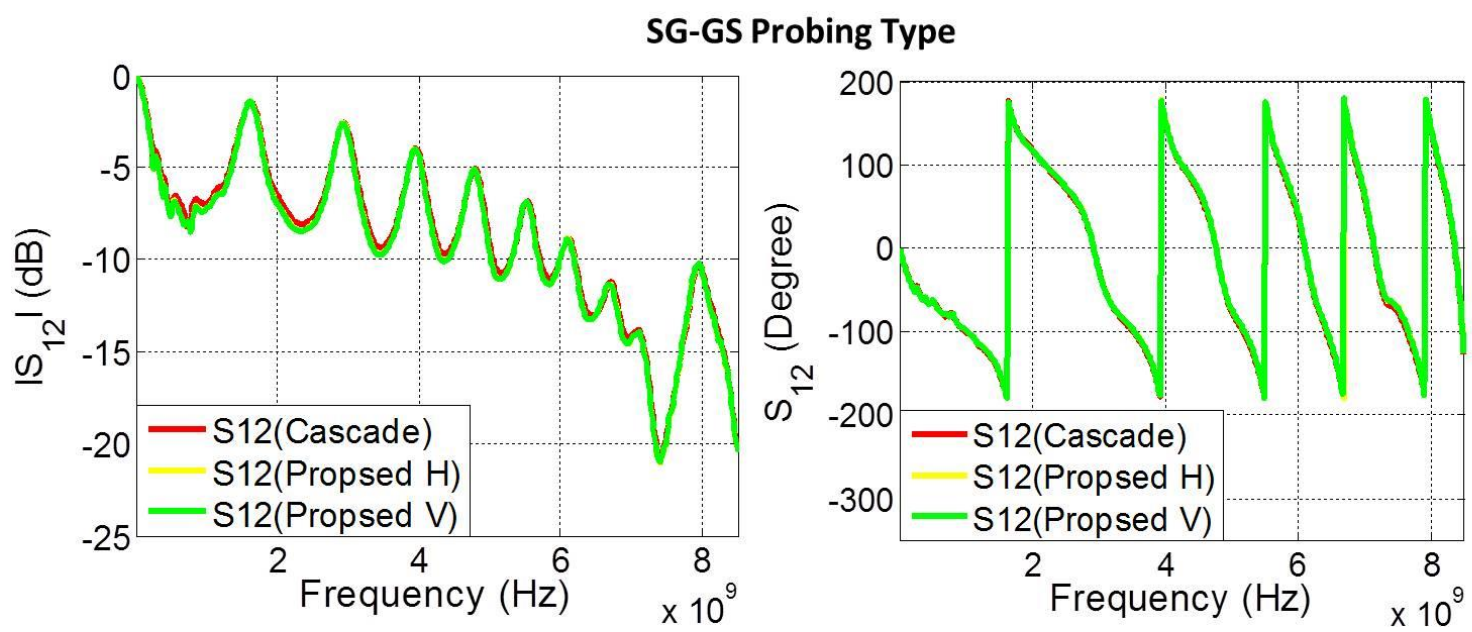

Figure 16. Insertion loss of meander lines measured after SG/GS calibration by commercial Cascade and 

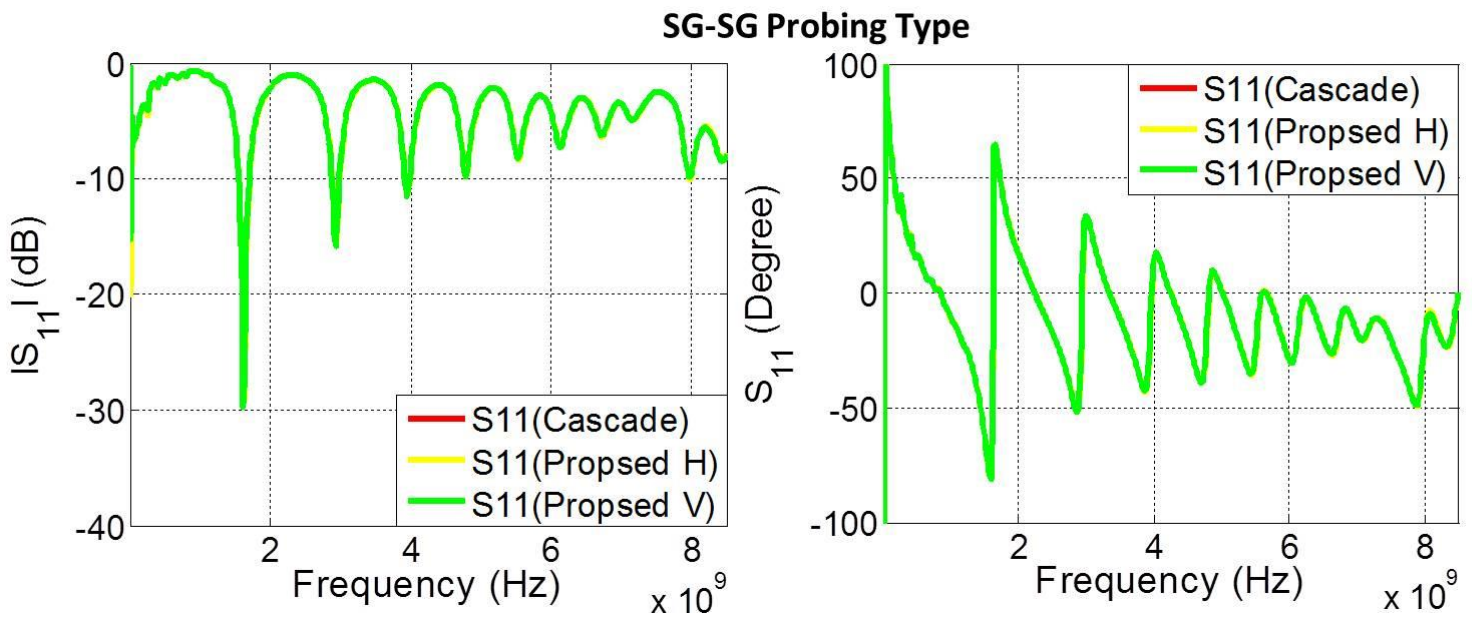

Figure 17. Return loss of meander lines measured after SG/SG calibration by commercial Cascade and

Proposed kit.

SG-SG Probing Type
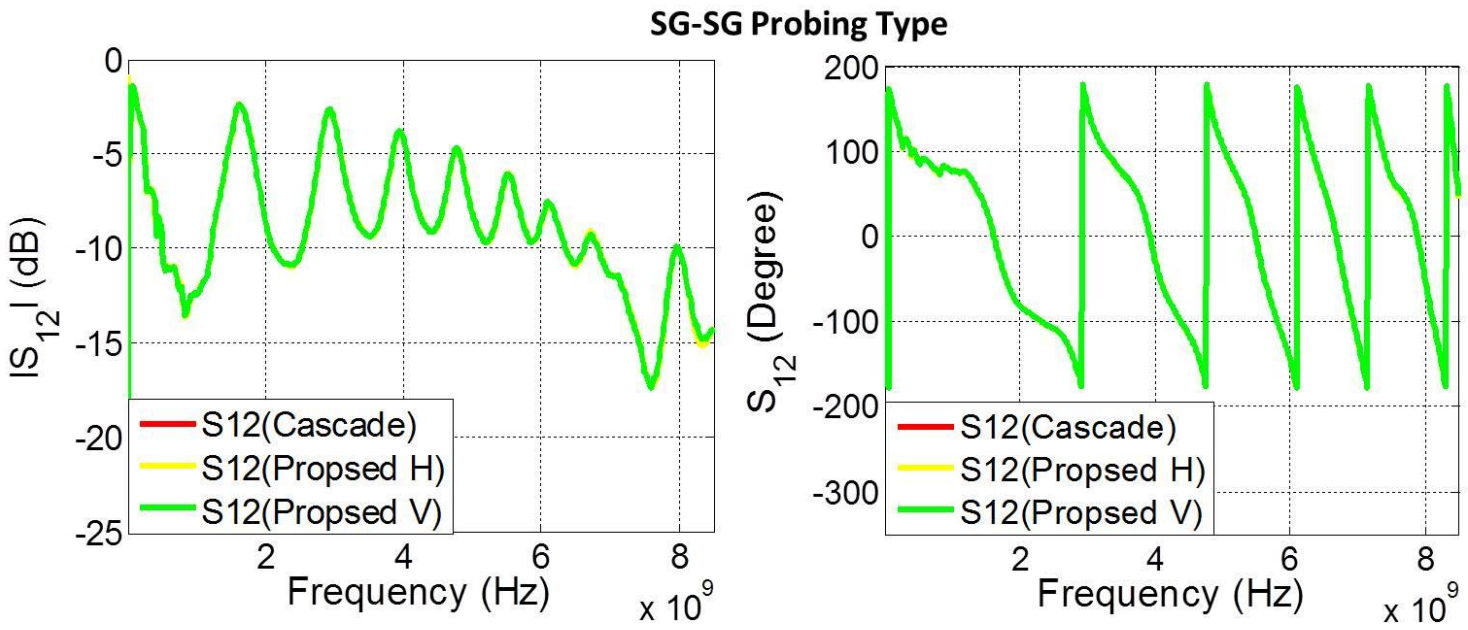

Figure 18. Insertion loss of meander lines measured after SG/SG calibration by commercial Cascade and

Proposed kit.

Not only the coplanar coupling happened in symmetrical meander lines, vertical coupling generated in via of PCB or TSV in silicon substrate are also widely existed [12][]. As mentioned previously, the conventional coplanar calibration kit is only able to provide a calibration in the same plane. Rotation of probes and location change of cables is inevitable. Thus, the accuracy would definitely be decreased because the probes and cables in the measurement of high speed signal are sensitive to the surrounding environment. A vertical interconnects such as via array as shown in the left part of Figure 19, the characterization of transmission and coupling effect require the probing on opposite planes as the port 1 and port2 in the right part of Figure 19. Proposed the vertical Thru in this manuscript can guarantee the accuracy up to $8 \mathrm{GHz}$ as discussed before, and maintain the location of probes and cables at the same time. In the via array, all vias are designed with the same size with a diameter of $0.2 \mathrm{~mm}$ and each side in via pad of $0.2 \mathrm{~mm}$. It makes the center to center distance of neighboring vias be $600 \mathrm{um}$.

Return loss results measured from the top plane for nearby two arrays, 4 via pairs at random locations are selected, and the return loss is the same as illustrated in the left one of Figure 21 . Coupling between any two via arrays varies with the distance between them. Increased distances from case 1 and case 6 were measured, and the coupling results were shown in the right part of Figure 21. The phenomena satisfy the physical theory, and it is also seen that the coupling reaches saturation when the distance goes up to value around $2000 \mathrm{um}$ for via arrays here. 

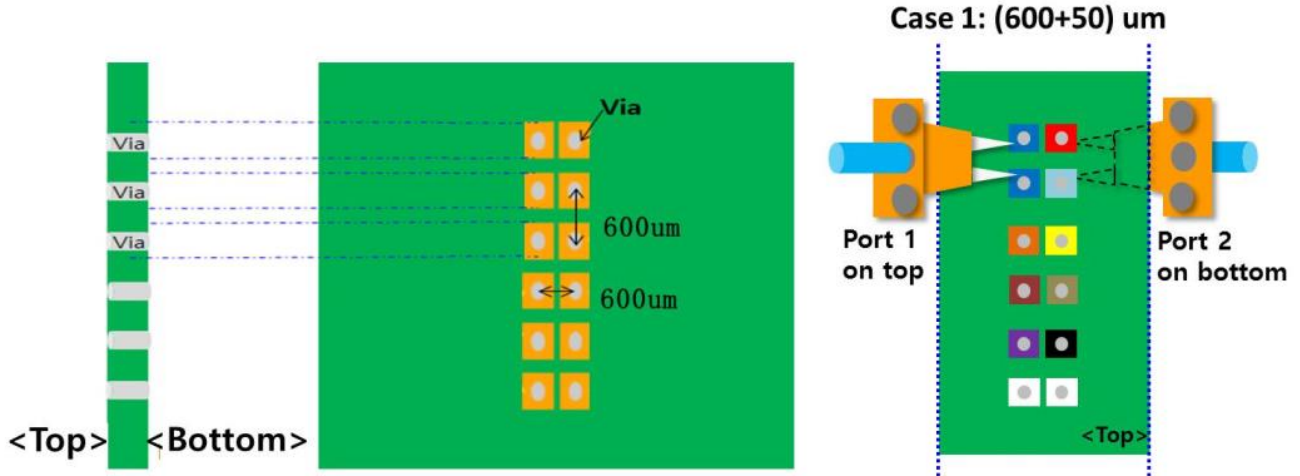

Figure 19. Measurement of coupling effect in via array on the basis of the proposed calibration kit.
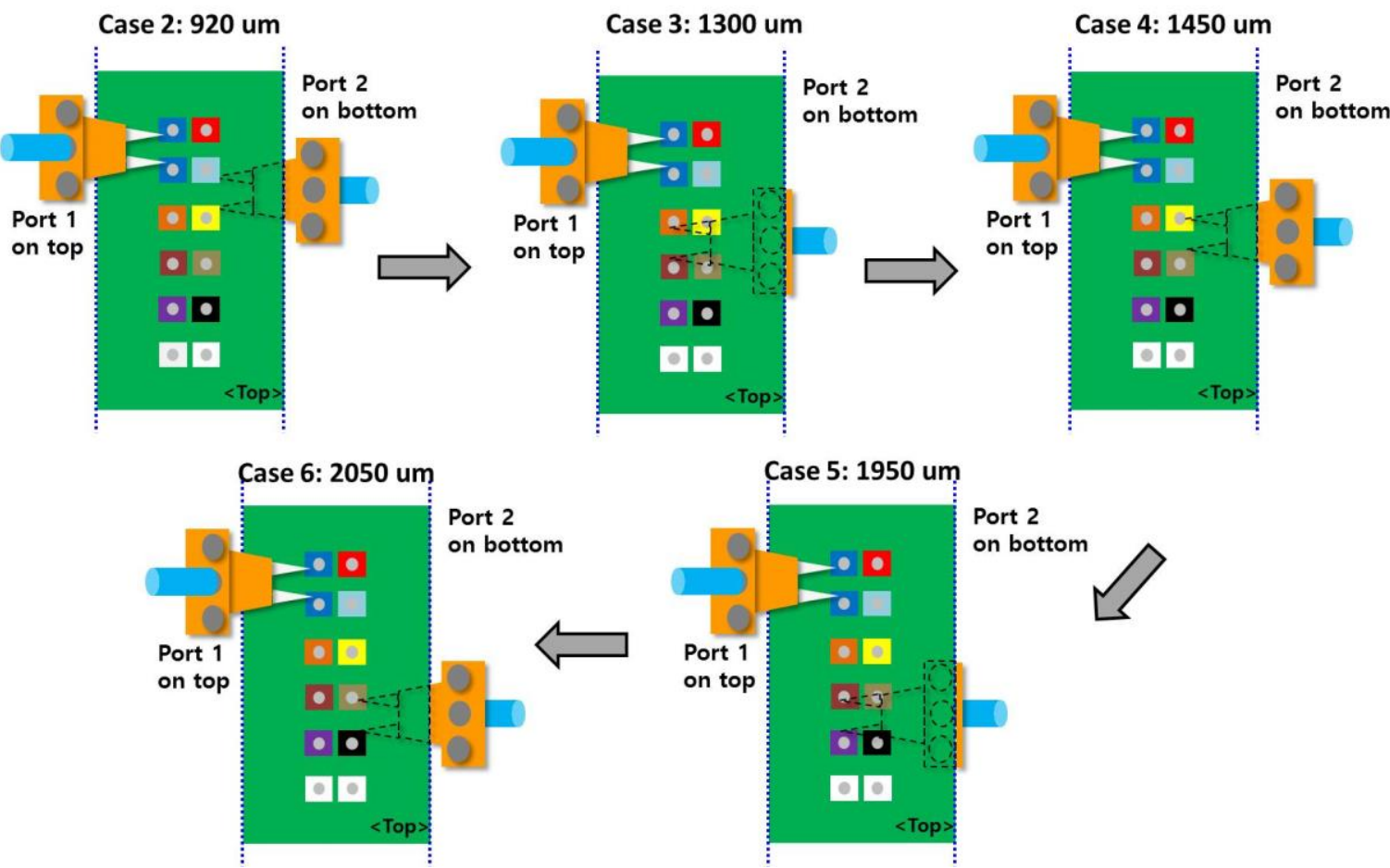

Figure 20. Measured couplings of via arrays in various distances.
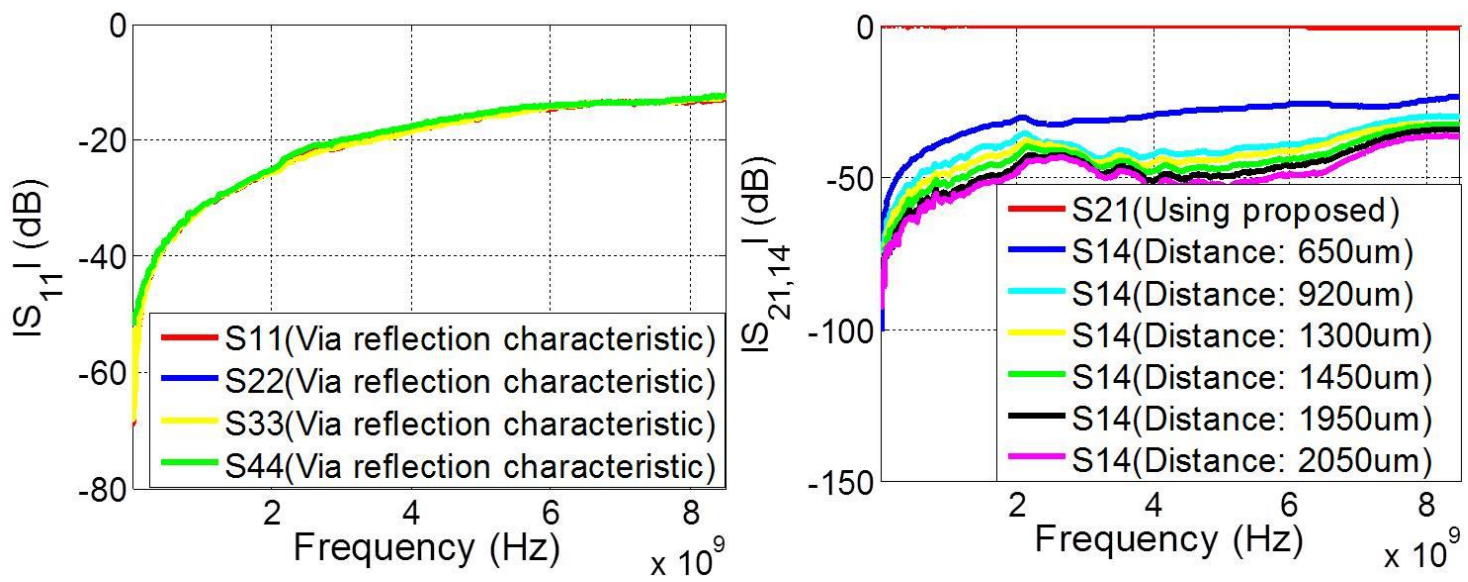

Figure 21. Measured coupling effect in via array on the basis of proposed calibration kit. 


\section{Conclusions}

A handy novel impedance standard substrate, aka calibration kit, for calibration on both horizontal and vertical interconnects by radio frequency and microwave probes are proposed. Unlike the conventional commercial calibration kit which only allows probing on the same plane, the proposed kit has a via structure that the probing on opposite plane is possible. Therefore, rotation of probe and location change of cables is not necessary on the basis of the novel kit when the calibration for vertical interconnects is required. Theoretic analysis and design in detail of the SOLT impedance standard substrate, especially the Thru case was demonstrated. The performance of the proposed kit was validated by the comparison of measurement reflection and transmission parameters after calibration using conventional and proposed kits. The proposed kit has a high accuracy from DC to $8 \mathrm{GHz}$. Two case studies were also discussed. One of them is the horizontal coplanar symmetrical meander line which calibration by both conventional and proposed kits is possible. Another case is the coupling effects between via arrays where the merit of our proposed kit is implemented and the coupling results were measured on the basis of the easy and convenient calibration.

\section{References}

1. J. H. Lau, Through-Silicon via for 3D Integration, New York, NY, USA:McGraw-Hill, 2013.

2. S. Pan and J. Fan, "Characterization of via structures in multilayer printed circuit boards with an equivalent transmission-line model", IEEE Trans. Electromagn. Compat., vol. 54, no. 5, pp. 1077-1086, Oct. 2012.

3. J. Kim, J. Cho, J. Kim, J.-M. Yook, J. C. Kim, J. Lee, et al., "High-Frequency Scalable Modeling and Analysis of a Differential Signal Through-Silicon Via", IEEE Trans. Compon. Packag. Manuf. Technol., vol. 4, no. 4, pp. 697-707, April 2014.

4. E.-P. LI, X. Wei, A. C. Cangellaris, E.-X. Liu, Y. Zhang, M. Damore, et al., "Progress Review of Electromagnetic Compatibility Analysis Technologies for Packages Printed Circuit Boards and Novel Interconnects", IEEE Trans. Electromagn. Compat., vol. 52, no. 2, pp. 248-265, May 2010.

5. B. Pu, K. Kim and W. Nah, "Package design methodology in consideration with signal integrity, power integrity and electromagnetic immunity," 2014 XXXIth URSI General Assembly and Scientific Symposium (URSI GASS), Beijing, 2014, pp. 1-4.

6. R. B. Marks, "A multiline method of network analyzer calibration", IEEE Trans. Microw. Theory Tech., vol. 39, no. 7, pp. 1205-1215, Jul. 1991.

7. U. Stumper, "Uncertainties of VNA S-parameter measurements applying the short-open-load-reciprocal (SOLR) calibration method", CPEM Conf. Dig., pp. 438-439, 2008.

8. J. Fleury and O. Benard, "Designing and characterizing TRL fixture Calibration standards for device modeling", Appl. Microw. Wirel., vol. 13, no. 10,2001, pp. 26-55.

9. Mostafa Emam. RF Characterization - The approach and the advantages, MOS-AK Tutorial Day Shanghai, June 26, 2016.

10. Pozar, David M. "Microwave engineering, 3rd." Danvers, MA: Wiley (2005).

11. B. Pu, K. H. Kim, S. Kim and W. Nah, "Modeling and Parameter Extraction of Coplanar Symmetrical Meander Lines", Electromagnetic Compatibility IEEE Transactions on, vol. 57, no. 3, pp. 375-383, June 2015.

12. S. Wu and J. Fan, "Analytical prediction of crosstalk among vias in multilayer printed circuit boards", IEEE Trans. Electromagn. Compat., vol. 54, pp. 413-420, Apr. 2012.

13. D. Seo, H. Lee, M. Park, and W. Nah, "Enhancement of Differential Signal Integrity by Employing a Novel Face Via Structure," IEEE Trans. Electromagn. Compat.,, vol. 60, no. 1, pp. 26-33, Feb. 2018. 